AperTO - Archivio Istituzionale Open Access dell'Università di Torino

\title{
Catastrophic antiphospholipid syndrome (CAPS).
}

\section{This is the author's manuscript}

Original Citation:

\section{Availability:}

This version is available http://hdl.handle.net/2318/142466

since

Published version:

DOI:10.1016/j.berh.2012.07.005

Terms of use:

Open Access

Anyone can freely access the full text of works made available as "Open Access". Works made available under a Creative Commons license can be used according to the terms and conditions of said license. Use of all other works requires consent of the right holder (author or publisher) if not exempted from copyright protection by the applicable law. 


\section{(3) \\ UNIVERSITÀ DEGLI STUDI DI TORINO}

This Accepted Author Manuscript (AAM) is copyrighted and published by Elsevier. It is posted here by agreement between Elsevier and the University of Turin. Changes resulting from the publishing process - such as editing, corrections, structural formatting, and other quality control mechanisms may not be reflected in this version of the text. The definitive version of the text was subsequently published in BAILLIERE'S BEST PRACTICE \& RESEARCH. CLINICAL RHEUMATOLOGY, Volume 26, Issue 4, August 2012, doi: 10.1016/j.berh.2012.07.005.

You may download, copy and otherwise use the AAM for non-commercial purposes provided that your license is limited by the following restrictions:

(1) You may use this AAM for non-commercial purposes only under the terms of the CC-BY-NC-ND license.

(2) The integrity of the work and identification of the author, copyright owner, and publisher must be preserved in any copy.

(3) You must attribute this AAM in the following format: Creative Commons BY-NC-ND license (http://creativecommons.org/licenses/by-nc-nd/4.0/deed.en), http://dx.doi.org/10.1016/j.berh.2012.07.005 


\section{Catastrophic Antiphospholipid syndrome (CAPS)}

Savino Sciascia ${ }^{1,2}$, Chary Lopez-Pedrera ${ }^{3}$, Dario Roccatello ${ }^{2}$, Maria Jose' Cuadrado ${ }^{3}$

1 Lupus Research Unit, The Rayne Institute, Division of Women's Health, King's College London, ${ }^{2}$ Centro di Ricerche di Immunopatologia e Documentazione su Malattie Rare (CMID), Università di Torino, ${ }^{3}$ IMIBIC-Reina Sofia Hospital and ${ }^{4}$ Louise Coote Lupus Unit, Guy’s and St Thomas’ NHS Foundation Trust, St Thomas' Hospital, London, UK

Correspondence:

Dr Savino Sciascia

Lupus Research Unit, The Rayne Institute, Division of Women's Health, King's College London, $4^{\text {th }}$ Floor Lambeth Wing, St Thomas' Hospital, London SE1 7EH

Tel: +44 02071883569

Fax: +4402076202658

Mail: savino.sciascia@unito.it

Conflict of interest

The authors declare no conflict of interest 


\begin{abstract}
Catastrophic Antiphospholipid syndrome (CAPS) is a very severe variant of the classic APS, characterised by clinical evidence of multiple organ involvement developing over a very short period of time, histopathological evidence of multiple small vessel occlusions, and laboratory confirmation of the presence of antiphospholipid antibodies (aPL), usually in high titre. Although patients with catastrophic APS represent less than $1 \%$ of all patients with APS, this is usually a life-threatening condition.
\end{abstract}

In this article we aimed to review the state-of-the art about current knowledge in pathogenesis, clinical manifestations, diagnosis, and treatment strategies in CAPS.

Key words: Catastrophic Antiphospholipid syndrome, Antiphospholipid syndrome, Anticoagulation, IVIG, Plasmapheresis 


\section{Introduction}

Catastrophic Antiphospholipid syndrome (CAPS) was first described by Asherson in 1992 [1]. It characterised by multiple vascular occlusive events, presenting over a short period of time, in patients with aPL positive. It is an uncommon presentation that occurs in $<1 \%$ of APS patients, often after a triggering factor such as anticoagulation withdrawal, surgery, minor surgical procedures, or infections. Mortality rate is around 50\% and treatment includes corticosteroids, anticoagulation, intravenous immunoglobulin and plasma exchange [2].

\section{Pathogenesis}

The etiopathogenesis of catastrophic APS remains incompletely understood. Several mechanisms have been proposed such as molecular mimicry, infections, activation of endothelium in the microvasculature and microvascular occlusions [3]. Kitchens et al [4] have suggested that the vascular occlusions are themselves responsible for the ongoing thrombosis. Clots continue to generate thrombin, fibrinolysis is impaired by an increase in plasminogen activator inhibitor type1 (PAI-1), and there is consumption of the natural anticoagulant proteins such as protein $\mathrm{C}$ and antithrombin. These multiple small-vessel occlusions cause extensive tissue necrosis which results in a systemic inflammatory response syndrome (SIRS), with excessive cytokine release from affected and necrotic tissues [5]. Proinflammatory cytokines, several products of the activated complement system (e.g. C3b, iC3b and C5a) and aPL themselves have each been demonstrated to activate endothelial cells, provide a stimulatory signal and upregulate adhesion molecules and tissue factor. These molecules can also act on leukocytes and platelets to increase their adhesion to vascular endothelium and to promote microthrombosis and the local release of toxic mediators, including proteases and oxygen-derived free radicals. The interaction between all 
these cells in the presence of aPL leads to the diffuse microvasculopathy that characterises CAPS and leads to multiorgan failure $[3,5,6]$.

\section{Clinical manifestations}

The most common known trigger for CAPS is infection. Other less common causes are anticoagulation withdrawal or low international normalized ratio (INR), medications (e.g oral contraceptive), obstetric complications, neoplasia, systemic lupus erythematosus (SLE) flares, trauma, and surgery. Nevertheless, in almost half of the cases, no obvious precipitating factors have been identified and CAPS can often occur in patients without any previous thrombotic history [2].

The clinical manifestations of CAPS depend on the organs that are affected, by the thrombotic events and the extent of the thrombosis, together with manifestations of the SIRS.

In contrast to classic APS, single venous or arterial medium to large blood vessel occlusions are uncommon in patients with catastrophic APS. Multiple organ dysfunction and failure, as a consequence of thrombotic microangiopathy, are responsible for the majority of the clinical features; however, large venous or arterial thrombosis can also occur in about one fifth of patients. In a review of 280 patients with CAPS [2] from the website based international CAPS registry, Cervera et al. reported that the first clinical manifestation at the time of the catastrophic episode was a pulmonary complication in $24 \%$ of the cases, a neurologic feature in $18 \%$ and a renal feature in $18 \%$. Although the initial presentation of CAPS may involve a single organ, in a very short period of time, typically days to weeks, patients develop clinical evidence of multiple organ thrombosis and dysfunction leading to organ failure that requires intensive care unit admission. In the same cohort of CAPS patients [2] during the catastrophic episode, 
intraabdominal involvement was identified in the majority of patients, mainly consists of renal (71\%), hepatic (33\%), gastrointestinal (25\%), splenic (19\%), adrenal (13\%), and pancreatic (8\%) manifestations. Intrathoracic involvement was also common and included pulmonary complications (64\%), mainly acute respiratory distress syndrome (ARDS) and pulmonary embolism, but occasionally intraalveolar hemorrhage, as well as cardiac manifestations (51\%), mainly cardiac failure and myocardial infarction or valve lesions. Cerebrovascular complications were also frequently present (62\%), mainly consists of encephalopathy and cerebrovascular accidents, but occasionally seizures, headache or silent brain infarcts. Skin manifestations were also frequent (50\%) with livedo reticularis, leg ulcers, necrotic lesions, digital gangrene, purpura, splinter haemorrhages, and multiple ecchymosis. Deep venous thrombosis (23\%) and peripheral arterial occlusive disease $(11 \%)$ were less frequently detected. Other lesions occasionally encountered were retinal involvement (7\%), mononeuritis multiplex (5\%) and bone marrow necrosis $(4 \%)$ [2].

\section{APS-related laboratory findings}

The most common APS related-laboratoy findings are thrombocytopenia, hemolytic anemia (often accompanied of schistocytes as in thrombotic microangiopathic hemolytic anemia), and disseminated intravascular coagulation (DIC). The presence of aPL, namely anticardiolipin (aCL), anti- $\beta 2$-glycoprotein I (anti- $\beta 2 \mathrm{GPI}$ ) and lupus anticoagulant (LA) is mandatory for the diagnosis. aPL are usually detected in high titre in CAPS patients. The role of other aPL, such as antiphosphatidylserine/prothrombin or anti-prothrombin in solid phase has been reported [7], but its role is still controversial.

\section{Diagnosis}


The diagnosis of CAPS can be challenging because of the acute onset of thrombosis at multiple levels with simultaneous dysfunction of different organs. The survival of the patients very much depends on an early diagnosis and treatment.

Preliminary CAPS classification criteria (Table 1) was proposed and agreed in Taormina, (Sicily) during the $10^{\text {th }}$ International Congress on aPL. Although these criteria are accepted for classification purposes, they might be a guide to a more consistent approach to the diagnosis [8]. An update of the diagnostic algorithm for CAPS was recently proposed [9] and discussed in 'Task Force on Catastrophic Antiphospholipid Syndrome (APS) and Non-criteria APS Manifestations' developed on the occasion of the 13th International Congress on Antiphospholipid, held in Galveston (Texas) in 2011[10]. This approach represents a step forward in diagnosis of CAPS but it needs to be validated versus other thrombotic microangiopathy.

To make the diagnosis of CAPS, there should be clinical evidence of multiple organ involvement developed over a short period of time, histopathologic evidence of multiple small-vessel occlusions and laboratory confirmation of the presence of aPL, usually in high titre. The positivity of aPL should be confirmed also later, when the acute clinical situation is over. aPL can be also positive in sepsis and other critical situations which share several clinical features with CAPS.

The differential diagnosis with sepsis, thrombotic thrombocytopenic purpura (TTP), hemolytic uremic syndrome (HUS), acute disseminated intravascular cogulation (DIC) and HELLP syndrome (hemolysis, elevated liver enzymes, and low platelets), has to be done. Some of the differences between these entities are summarised in Table 2.

CAPS resembles severe sepsis in its presentation. Both share the symptoms of SIRS [temperature $>38^{\circ} \mathrm{C}$, tachycardia, increased respiratory frequency, hypotension, elevated white blood cell count or with $>10 \%$ immature forms (band)]. Infection is the most common trigger factor of CAPS and, 
sometimes, both situations can co-exist. An aggressive treatment of the infection can markedly improve the prognosis.

TTP, characterised by thrombocytopenia and microangiopathic haemolytic anemia, also share with CAPS the presence of renal dysfunction and neurological features. Microangiopathic haemolytic anemia consists in a nonimmune hemolysis (direct antiglobulin test negative) together with the presence of red cell fragmentation (schistocytes), in peripheral blood smear. The presence of more than $1 \%$ of schistocyte strongly suggests the diagnosis of TTP (11).

DIC causes multiple thrombosis and haemorrhage. Its features also include renal, liver, lung and central nervous system involvement. The presence of widespread haemorrhage is not so frequent in CAPS.

\section{Treatment}

The optimal treatment regime in CAPS is unknown. Current treatment guidelines suggest, in addition to early diagnosis, aggressive therapies to avoid the potentially fatal outcome. The combination of high doses of intravenous (i.v.) heparin, i.v. steroids, i.v. immunoglobulins and/or repeated plasma exchanges are the basic treatment of choice for all patients with this severe condition (Evidence Level II) [10].

I.v. heparin is usually administrated during the critical period. When the patient is stable, oral anticoagulation with an INR target around 3.0 can be initiated. Heparin is discontinued when the target INR is achieved with warfarin. The beneficial effects of Heparin are mediated by its capacity to inhibit thrombin, factor Xa and other activated clotting factors [11]. Heparin also inhibits complement activation [12]. Nevertheless, this mechanism has not been proven in humans, but in the setting of CAPS where there is an important inflammatory component, might be relevant. 
Corticosteroids are recommended in a situation with SIRS and excessive cytokine release, as they are able to inhibit the proinflammatory status mediated by both the NF-kB and mitogen-activated protein kinase pathway [13].

When initiated promptly plasma exchange (PE) can be considered an effective and safe treatment for CAPS. It is useful by removing pathological aPL, cytokines and complement and also incorporating natural anticoagulants like antithrombin and protein C. The treatment of CAPS with PE is not well standardized. The best replacement fluid for PE is still a controversial issue [14]. However, the efficacy of PE has been shown in other thrombotic microangiopathy and since CAPS is a critical situation with a high rate of mortality, it is recommended as part of the CAPS treatment [15].

The rationale to use IVIG in the treatment of CAPS is based on its capacity to block pathological antibodies, increases its clearance, acts on the complement system and supresses cytokines [16]. Their use in refractory cases of APS resistant to standard therapy has also recently reported [17]. IVIG should be specially indicated in situations where there is concomitant infection because of its role as immunomodulator rather than immunosuppressor [18].

Immunosuppressive drugs such as i.v. cyclophosphamide might be helpful in patients with active systemic autoimmune disease mainly SLE and systemic vasculitis but its use in CAPS is not associated with an improvement in the survival rate [19].

Some case reports about the use of rituximab - an anti-CD20 monoclonal-in the treatment of CAPS have been published [20-22] . Although more data are necessary to support the use of this drug in the setting of CAPS, current experience seems quite promising, especially in patients with severe thrombocytopenia. More recently, a case of induction of durable remission in recurrent CAPS was obtained via inhibition of terminal complement with eculizumab [23]. 
As patients with CAPS have a life-threating condition and high mortality, they need adequate management in ICU that should include hemodialysis, mechanical ventilation or cardiovascular support for shock. Other factors like aggressive treatment of infection, debridement or amputation of necrotic tissues/organs, careful management of intravascular instrumentation, especially arterial which can lead to new clots, are extremely important and can substantially improve the rate of survival.

\section{Prognosis}

The mortality has been considerably reduced (from more than $50 \%$ to $30 \%$ ) over the past 10 years by the use of the recommended therapies. The use of the combination of anticoagulation, corticosteroids, plasma exchange and intravenous immunoglobulin (IVIG) has been associated with the highest rate of survival [2].

Data addressing the long-term follow-up of patient with one episode of CAPS are scanty [24]. A review including 136 published patients with CAPS showed that 63/136 (46\%) died at the time of the event. Seventy three patients survived but information was available only from 58 (79\%). Thirty-eight of $58(66 \%)$ patients did not develop any other thrombotic event during a medium follow-up time of 67.2 months. Fifteen of 58 (26\%) patients developed further APS related thrombosis after the initial catastrophic APS event and the mortality rate of these patients was about $25 \%$ (4/15 patients). In summary, $66 \%$ of survivors remained thrombosis free and $17 \%$ developed further APS-related thrombosis. None of the patients developed further catastrophic APS.

Comparing the demographic, clinical, and immunologic characteristics of patients who survived to those who died, older age, pulmonary and renal involvement, the presence of SLE and high titre of antinuclear antibodies (ANA) were associated with a higher mortality rate [25] 
There is few data regarding CAPS relapse. Three patients with seven episodes of CAPS have been described. The median time between the episodes was 12.5 months (range, 2.5-48). From the clinical point of view, the most significant manifestations were renal involvement (present in 5 episodes), followed by central nervous system and cardiac involvement (4 episodes each), and pulmonary and hepatic involvement ( 3 episodes each). Interestingly, thrombocytopenia (platelet count less than $50 \times 10^{9} / \mathrm{L}$ ) and red cell fragmentation (schistocytes) were reported in all 7 episodes [26]. In the CAPS registry, a total of 18 episodes of relapse in eight patients, have been described [22]. Overall, the precipitating factors in these patients were infection, subtherapeutic anticoagulation level and anticoagulation withdrawal. The symptoms were very similar to the first CAPS episode (renal failure followed by cerebral, cardiac, and pulmonary involvement). Thrombocytopenia was present in 17 patients and hemolytic anemia in 13. LA test was the most common aPL positive [19]

In conclusion, every physician managing patients with multiorgan failure should be aware of the possibility of CAPS. This diagnosis should be strongly suspected in patients previously diagnosed with APS or any underlying systemic autoimmune disease. A prompt diagnosis will enable physicians to take measures to prevent death from this syndrome. An aggressive treatment with steroids, anticoagulation and IVIG in an ICU setting, will help prevent the progression of organ failure or the development of septic shock in the infected patients. 


\section{Summary}

Catastrophic Antiphospholipid syndrome (CAPS) is a very severe variant of the classic APS, characterised by clinical evidence of multiple organ involvement developing over a very short period of time, histopathological evidence of multiple small vessel occlusions, and laboratory confirmation of the presence of antiphospholipid antibodies (aPL), usually in high titre. Although patients with catastrophic APS represent less than $1 \%$ of all patients with APS, this is usually in a life-threatening situation. The mortality has been considerably reduced (from more than $50 \%$ to $30 \%$ ) over the past 10 years by the use of the recommended therapies (anticoagulation, corticosteroids, plasma exchange and IVIG). Relapsing cases still represent a challenge for treating physicians. 
1. Evidence of involvement of 3 organs, systems, and/or tissues

2. Development of manifestations simultaneously or in less than 1 week

3. Confirmation by histopathology of small vessel occlusion in at least 1 organ/tissue

4. Laboratory confirmation of the presence of aPL (LAC and/or aCL and/or anti-2GPI antibodies)

Definite CAPS:

- All 4 criteria

Probable CAPS:

- All 4 criteria, except for involvement of only 2 organs, system, and/or tissues

- All 4 criteria, except for the absence of laboratory confirmation at least 6 weeks apart associable to the early death of a patient never tested for aPL before onset of CAPS)

- 1,2 , and 4

- 1, 3, and 4, and the development of a third event in $>1$ week but $<1$ month, despite anticoagulation treatment 
Table 2. Differential diagnosis of catastrophic antiphospholipid syndrome

\begin{tabular}{|l|c|c|c|c|c|}
\hline & Fibrinogen & $\begin{array}{c}\text { Hemolytic } \\
\text { anemia }\end{array}$ & Schistocytes & Thrombocytopenia & aPL \\
\hline CAPS & Normal & $+/-$ & $+/-$ & $+/-$ & ++ \\
\hline Sepsis & Normal/low & + & $+/-$ & $+/-$ & $+/-$ \\
\hline TTP-HUS & Normal & + & ++ & ++ & - \\
\hline DIC & Low & $+/-$ & $+/-$ & + & - \\
\hline
\end{tabular}

DIC: Disseminated intravascular coagulation; TTP-HUS: thrombotic thrombocytopenic purpura hemolytic-uremic syndrome 


\section{References}

[1] Asherson RA. The catastrophic antiphospholipid syndrome. The Journal of rheumatology. 1992;19:508-12.

[2] Cervera R, Bucciarelli S, Plasin MA, et al. Catastrophic antiphospholipid syndrome (CAPS): descriptive analysis of a series of 280 patients from the "CAPS Registry". Journal of autoimmunity. 2009;32:240-5.

[3] Ortega-Hernandez OD, Agmon-Levin N, Blank M, et al. The physiopathology of the catastrophic antiphospholipid (Asherson's) syndrome: compelling evidence. $\underline{\text { Journal of }}$ autoimmunity. 2009;32:1-6.

[4] Kitchens CS. Thrombotic storm: when thrombosis begets thrombosis. The American journal of medicine. 1998;104:381-5.

[5] Vora SK, Asherson RA, Erkan D. Catastrophic antiphospholipid syndrome. Journal of intensive care medicine. 2006;21:144-59.

[6] Bhatia M, Moochhala S. Role of inflammatory mediators in the pathophysiology of acute respiratory distress syndrome. The Journal of pathology. 2004;202:145-56.

[7] Hirakawa E, Saito K, Hirata S, et al. A case of catastrophic antiphospholipid antibody syndrome complicated with systemic lupus erythematosus, double positive for anticardiolipin/beta(2) glycoprotein I and anti-phosphatidylserine/prothrombin autoantibodies. Modern rheumatology/ the Japan Rheumatism Association. 2011.

[8] Asherson RA, Cervera R, de Groot PG, et al. Catastrophic antiphospholipid syndrome: international consensus statement on classification criteria and treatment guidelines. Lupus. 2003;12:530-4.

[9] Erkan D, Espinosa G, Cervera R. Catastrophic antiphospholipid syndrome: updated diagnostic algorithms. Autoimmunity reviews. 2010;10:74-9. 
[10] Cervera R, Tektonidou MG, Espinosa G, et al. Task Force on Catastrophic Antiphospholipid Syndrome (APS) and Non-criteria APS Manifestations (I): catastrophic APS, APS nephropathy and heart valve lesions. Lupus. 2011;20:165-73.

[11] Weitz JI, Hirsh J, Samama MM. New anticoagulant drugs: the Seventh ACCP Conference on Antithrombotic and Thrombolytic Therapy. Chest. 2004;126:265S-86S.

[12] Girardi G, Redecha P, Salmon JE. Heparin prevents antiphospholipid antibody-induced fetal loss by inhibiting complement activation. Nature medicine. 2004;10:1222-6.

[13] Auphan N, DiDonato JA, Rosette C, et al. Immunosuppression by glucocorticoids: inhibition of NF-kappa B activity through induction of I kappa B synthesis. Science. 1995;270:286-90.

[14] Marson P, Bagatella P, Bortolati M, et al. Plasma exchange for the management of the catastrophic antiphospholipid syndrome: importance of the type of fluid replacement. $\underline{\text { Journal of }}$ internal medicine. 2008;264:201-3.

[15] Espinosa G, Bucciarelli S, Cervera R, et al. Thrombotic microangiopathic haemolytic anaemia and antiphospholipid antibodies. Annals of the rheumatic diseases. 2004;63:730-6.

[16] Lee SJ, Chinen J, Kavanaugh A. Immunomodulator therapy: monoclonal antibodies, fusion proteins, cytokines, and immunoglobulins. The Journal of allergy and clinical immunology. 2010;125:S314-23.

[17] Sciascia S, Giachino O, Roccatello D. Prevention of thrombosis relapse in antiphospholipid syndrome patients refractory to conventional therapy using intravenous immunoglobulin. $\underline{\text { Clinical }}$ and experimental rheumatology. 2012.in press

[18] Hartung HP, Mouthon L, Ahmed R, et al. Clinical applications of intravenous immunoglobulins (IVIg)--beyond immunodeficiencies and neurology. Clinical and experimental immunology. 2009;158 Suppl 1:23-33. 
[19] Bucciarelli S, Espinosa G, Cervera R, et al. Mortality in the catastrophic antiphospholipid syndrome: causes of death and prognostic factors in a series of 250 patients. Arthritis and rheumatism. 2006;54:2568-76.

[20] Rubenstein E, Arkfeld DG, Metyas S, et al. Rituximab treatment for resistant antiphospholipid syndrome. The Journal of rheumatology. 2006;33:355-7.

[21] Iglesias-Jimenez E, Camacho-Lovillo M, Falcon-Neyra D, et al. Infant with probable catastrophic antiphospholipid syndrome successfully managed with rituximab. Pediatrics. 2010;125:e1523-8.

[22] Espinosa G, Berman H, Cervera R. Management of refractory cases of catastrophic antiphospholipid syndrome. Autoimmunity reviews. 2011;10:664-8.

[23] Shapira I, Andrade D, Allen SL, Salmon JE. Induction of durable remission in recurrent catastrophic antiphospholipid syndrome via inhibition of terminal complement with eculizumab. Arthritis and rheumatism. 2012.in press

[24] Erkan D, Asherson RA, Espinosa G, Cervera R, Font J, Piette JC, et al. Long term outcome of catastrophic antiphospholipid syndrome survivors. Annals of the rheumatic diseases. $2003 ; 62: 530-3$.

[25] Bayraktar UD, Erkan D, Bucciarelli S, Espinosa G, Asherson R. The clinical spectrum of catastrophic antiphospholipid syndrome in the absence and presence of lupus. The Journal of rheumatology. 2007;34:346-52.

[26] Asherson RA, Espinosa G, Menahem S, Yinh J, Bucciarelli S, Bosch X, et al. Relapsing catastrophic antiphospholipid syndrome: report of three cases. Seminars in arthritis and rheumatism. 2008;37:366-72. 
